\title{
Program Detection in Wireless Feeler Networks
}

\author{
D.Jeyapriya S.Theivasigamani, R.Velvizhi, P.Nandhini
}

\begin{abstract}
Arrangement of Wireless Sensor Networks (WSN) has achieved huge advancement of late making it wrap up doubtlessly outstanding amongst other making locales of as far as possible correspondences structure. The basic issue in WSN is the prerequisite on essentialness and planning resources limit in bit which diminishes down potential value of sensor center. Similarly constant usage of a lone briefest course among source and sink realizes a shorter future of a sensor center point which hence spoils the framework execution. The basic idea for the wide scale WSN is the development on control use efficiency. Thusly grouping is basic essentialness gainful controlling methodology for WSN. The proposed Spherical Routing show (SpRP) are arranged and its execution estimations are evaluated as for example number of group transmitted, number of package got, rate in bundle transport, throughput and typical waiting imperativeness and differentiated and the execution estimations of Modified LEACH (M-LEACH) grouping Protocol. The conclusion gathering Protocol. The end from the examination of results is that SpRP show achieves better streamlined execution contrast to celebrated M-LEACH show. The foreseen work results from the execution estimations can incorporates extra life time in a WSN to give unparalleled Quality of Service
\end{abstract}

Keywords: Wireless, sensor, networks.

\section{I.INTRODUCTION}

Watchwords - Energy; Data Capacity; Spherical Routing; M-LEACH; Life traverse; Quality of Service.In Wireless Sensor Network (WSN) a few remote sensors hubs are conveyed over the wide geological region where every hub capacity is to detect info credits encompassing to its condition. For transmitting the valuable message to the base station sensor hubs build a streamlined most limited way utilizing middle of the road sensor hubs. Every Sensor Nodes facilitated to its neighbor sensor hubs for fruitful directing of data[2]. There are wide verities of existing directing plan which improves best shower between source hubs to goal hub. Generally Most of the routing methods adopt the approach which uses best routing metrics for efficient energy and resource utilization group heads, with the goal that the power scattering amid correspondence considering the base station is stretch out to all sensor hubs[3]. Drain utilizes one

Revised Manuscript Received on June 22, 2019.

D.Jayapriya, Department of CSE ,Bharath Institute of Higher Education and Reaesrch,Chennai,Thamilnadu,India.

S.Deivasigamani ,Deparrtment of CSE, Bharath Institute of Higher Education and Research Chenai,Thamilnadu ,India.

R.Velvizhi , Department of CSE ,Bharath Institute of Higher Education and Reaesrch,Chennai,Thamilnadu,India.

P.Nandhini ,Deparrtment of CSE, Bharath Institute of Higher Education and Research Chenai,Thamilnadu ,India. bounce steering with every hub ready to pass on message specifically to the group head and the base station. Along these lines, this strategy isn't reasonable to systems in the utilization of vast scope region. What's more, the outline of dynamic bunching incorporates all the more overhead, for example, changes in head, promotions and so on., which may debilitate the pick up in control utilization.

\section{MATERIALS AND METHODOLOGY}

II. GBDD (Grid Based Data Dissemination) Framework cell zone is shaped by twin radio scope of a bit. In GBDD lattice building process begins relying upon sink which was first associated with correspondence. Course metric are taken after as when if substantial lattice is available, sink discovers closest spot hub. The advantage of this convention is that it ensures continuous information discharge from source hubs to base station. In any case, it devours more power when the rate is high.

\section{ARA (Adaptive Routing Algorithm)}

The system presence is enhanced by considering the sensor hub exceptional vitality and it makes a versatile course way which is based versatile steering calculation helpful for network structure of WSN[4]. The reenactment result shows the square sensor organize offers key advantages difference to the hexagonal sensor arrange, despite the fact that it has more scope surface. ARA plot is likewise most loved for self-assertively conveyed sensor hubs. The hubs are framed in bunches and the groups are masterminded in matrix topologies. Along these lines the bunch head can be considered as a hub from the network.

IV. GBDAS (Grid-Based Data Aggregation Scheme) Convention divided the sensors ground into a framework of cells to advance information transmission to the Base Station. In message gathering round, part hubs alternate to be cell head. In like manner, cell heads in the fasten likewise alternate to be the chain pioneer[1]. Along these lines, the power utilization of sensor hubs is equally conveyed in order to abuse their lifetimes. Thus, the life expectancy of the entire WSN expands.

\section{V.CBDAS (Cycle-Based Data Aggregation Scheme)} Hub discover which cell it identified with by a straightforward number juggling process. Matrix based WSN construct the network framework by isolating the entire geological segment into a lattice of cells. Better lifetime is accomplished with of cells. Better lifetime is practiced with this arrangement of the sensor center points to upgrade the future of the whole

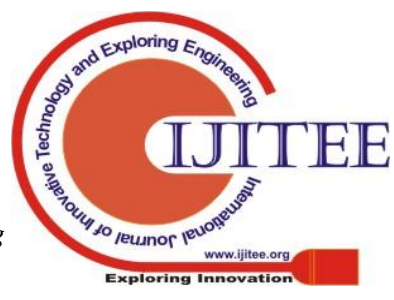


sensor mastermind. This methodology beats the other grid based methodology to the extent improving power level, estimation of the framework, and conservativeness of the center.

\section{TTDD (Two-Tier Data Dissemination)}

Gives distinctive compact base stations data transport. Tolerating that sensor centers are as yet and position careful, each datum source proactively manufactures a network topology for spreading data to the compact sinks. Furthermore, sensor center points are territory careful and stationary, while base station may move their regions vivaciously depending upon situation. The reenactment result shows that TTDD perform landing of data from numerous sources to various versatile sinks with cutting edge execution .

It gets data from the limit segment and plays out the computational endeavor. ROM and RAM .It is used for securing information. Trans-gatherer Transmission and Reception of information. Sensor(s) ,To distinguish input properties. Battery WSN center portions draw essentialness from it. Sensor center points are used as a piece of a couple of uses with perfect occupations of WSN center imperativeness. The use of WSN are regularly immaculate in watching framework, biological, coursed control structure, radioactive sources distinguishing proof, green and develop, web, shield and perception.

WSN is fit for being used for huge scale an area media transmission linkage transversely over different land areas. The arrangement and sending of WSN transversely over various separate pieces of wide region and their interconnectivity should be conceivable and extended for the covering the area of completion earth by game plan of social affair of frameworks which is approximated into round shape .. Globe scope structure utilizing WSN ${ }^{e e} \mathrm{~s}$

\section{RESULTS AND DISCUSSIONS}

The pervasive Grid based Routing Techniques are investigated to grasp the present guiding strategies in WSN and these surveys papers focal points have exhibited propelled results to improve the framework lifetimes stand out from various sorts WSN coordinating plans.Sensor Channel (Low Energy Adaptive Clustering Hierarchy) is the for the most part recognized different leveled guiding system . Sensor center point organizes each other to help in structure a round guiding association between source centers to base station by with the way which is comparable to a logarithmic round bend inverse route to the notice way. For the asset obliged remote sensor hubs logarithmic round bend is picked which is perfect for SpRP. It is expected that ahead of time the circular bend related required parameters are in earlier introduced to each sensor hub. At the point when sensor hubs watch a few occasions and prepared to get scattered, it starts the circular spread. The picking of the following jump neighbor hub relies upon the utilization of Spherical Path Search Protocol (SPSP) which fits the arranged best round way and after that to the picked sensor hub it sends an ad which incorporates the position and ID of the first bounce hub, the circular course of the former jump hub, and some more parameters like commercial TTL (Time-To-Live), the most extreme bounce number of the dispersion way, and so forth.

\section{CONCLUSIONS}

In the wake of getting a commercial bundle by a sensor hub, it stores a nearby duplicate of the notice message, at that point make utilization of an indistinguishable SPSP to choose a neighbor from the consequent bounce, and afterward exchange the ad message. By this approach, sending of the ad message happens jump by bounce in the sensor organize which takes after a circular like track. At the point when the circular connection achieves the edge, or the when confinement on jump is achieved directing will stop when it meets some preset condition. Inverse way question strategy is like the spread technique. At the point when a question is started by a sink hub, the inquiry takes after the contrary way round way till it fulfill the circular scattering, or the end condition is satisfied. The source hub can now and again raise to date the data along the circular way, or work in an unforeseen frame that begins the round appropriation each time the concerned occasion is watched. At ith jump SPSP utilizes way seek calculation which is appeared by the accompanying condition:

\section{REFERENCES}

W.R.Heinzelman,A.Chandrakasan, and H. Balakrishnan, "Energy efficient communication protocol for wireless micro sensor networks," in Proceedings of the 33rd Annual Hawaii International Conference on System Sciences (HICSS), pp. 10-20, January 2000.

2. S.Lindsey and C. S. Raghavendra, "PEGASIS: Power-efficient gathering in sensor information systems," in Proceedings of the IEEE Aerospace, vol. 3, pp. 1125-1130, 2002

3. Manjeshwar, D.P. Agrawal, "TEEN: a protocol for enhanced efficiency in wireless sensor networks," in Proceedings of the 1 st International Workshop on Parallel and Distributed Computing Issues in Wireless Networks and Mobile Computing, San Francisco, CA, April 2001.

4. Manjeshwar and D. P. Agrawal, “APTEEN: a hybrid protocol for efficient routing and comprehensive information retrieval in wireless sensor networks," in Proceedings of the 2nd International Workshop on Parallel and Distributed Computing Issues in Wireless Networks and Mobile computing,

\section{AUTHORS PROFILE}

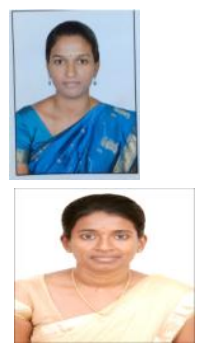

D.Jayapriya , Department of CSE ,Bharath Institute of Higher Education and Reaesrch,Chennai,Thamilnadu,India.

S.Theivasigamani ,Deparrtment of CSE, Bharath Institute of Higher Education and Research Chenai,Thamilnadu ,India

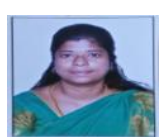

R.Velvizhi ,Deparrtment of CSE, Bharath Institute of Higher Education and Research Chenai,Thamilnadu ,India

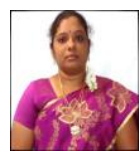

P.Nandhini ,Deparrtment of CSE, Bharath Institute of Higher Education and Research Chenai,Thamilnadu ,India 